University of Nebraska - Lincoln

DigitalCommons@University of Nebraska - Lincoln

Biological Systems Engineering: Papers and

Publications

Biological Systems Engineering

$5-2004$

\title{
Transdermal delivery of phosphorodiamidate Morpholino oligomers across hairless mouse skin
}

\author{
Angela K. Pannier \\ University of Nebraska-Lincoln, apannier2@unl.edu \\ Vikram Arora \\ AVI BioPharma, Corvallis, OR \\ Patrick L. Iversen \\ AVI BioPharma, Corvallis, OR \\ Rhonda M. Brand \\ Feinberg School of Medicine at Northwestern University, rhbrand@enh.org
}

Follow this and additional works at: https://digitalcommons.unl.edu/biosysengfacpub

Part of the Biological Engineering Commons

Pannier, Angela K.; Arora, Vikram; Iversen, Patrick L.; and Brand, Rhonda M., "Transdermal delivery of phosphorodiamidate Morpholino oligomers across hairless mouse skin" (2004). Biological Systems Engineering: Papers and Publications. 159.

https://digitalcommons.unl.edu/biosysengfacpub/159

This Article is brought to you for free and open access by the Biological Systems Engineering at DigitalCommons@University of Nebraska - Lincoln. It has been accepted for inclusion in Biological Systems Engineering: Papers and Publications by an authorized administrator of DigitalCommons@University of Nebraska Lincoln. 


\title{
Transdermal delivery of phosphorodiamidate Morpholino oligomers across hairless mouse skin
}

\author{
Angela K. Pannier, ${ }^{1}$ Vikram Arora, ${ }^{2}$ Patrick L. Iversen, ${ }^{2}$ and Rhonda M. Brand ${ }^{1,3}$
}

1. Department of Biological Systems Engineering, University of Nebraska-Lincoln, Lincoln, NE 68583-0726

2. AVI BioPharma, 4575 SW Research Way \#200, Corvallis, OR 97333

3. Department of Internal Medicine, Division of Emergency Medicine Evanston Northwestern Healthcare, Feinberg School of Medicine at Northwestern University, 1001 University Place, Evanston, IL 60201

Corresponding author - R. M. Brand, tel 224 364-7688, fax 224 364-8011, e-mail rhbrand@enh.org

\begin{abstract}
The skin is the largest organ in the body and an obvious route for both local and systemic drug delivery. Antisense oligomers have tremendous potential as therapeutic agents for numerous diseases. The objective of this study was to investigate the influence of vehicle on the transdermal delivery of several phosphorodiamidate Morpholino oligomers (PMOs) with different sizes, lengths, base compositions, sequences, and lipophilicities. Eleven different PMOs were synthesized complementary to biologically relevant gene targets and delivered across hairless mouse skin in vitro using vehicles composed of $95 \%$ propylene glycol, $5 \%$ linoleic acid (PG/LA), water, $50 \%$ water:50\% PG/LA, and 75\% water:25\% PG/LA. The data suggest that size, sequence and guanine composition all influence transdermal penetration. There was an inverse linear relationship between size and penetration for a given sequence when the PG/LA formulation was used $\left(r^{2}=0.94\right)$, but this trend was not evident when the vehicle contained water. An oligomer targeted to the gene p53 had lower than expected transdermal penetration based on its size, but was shown to localize within the skin, demonstrating that sequence and thus target will impact transdermal delivery. The presence of G-quartets correlated with better PMO penetration from a water vehicle. Overall, the data suggest that some oligomers and vehicles would be better for transdermal delivery and others for topical applications.
\end{abstract}

Keywords: transdermal drug delivery, antisense, oligonucleotides, penetration enhancers, hairless mouse skin

\section{Introduction}

Antisense oligomers represent the ultimate in rational drug design, in that they have been developed to block the translation of a particular protein through specific interaction with its mRNA. Phosphorodiamidate Morpholino oligomers (PMOs) represent a neutral DNA analog chemistry, in that, they have a non-ionic phosphorodiamidate backbone combined with a morpholine ring replacing the deoxyribose sugar with the additional replace- ment of one of the non-bridging oxygen atoms with a dimethylamine (Summerton and Weller, 1997). These modifications confer improved oligomer properties, including better hybrid duplex stability, little or no non-antisense activity, relatively good aqueous solubility, low production costs (Summerton and Weller, 1997), resistance to nucleases (Hudziak et al., 1996), high specificity and better efficacy than other types of antisense structural types (Stein et al., 1997). With their neutral chemistry, PMOs have the potential to be delivered passively, with 
chemical penetration enhancers such as water or propylene glycol.

Transdermally delivered oligomers with charged backbones have been demonstrated to cross the skin in vitro (Nolen et al., 1994; Brand and Iversen, 1996; Brand et al., 1998a; Oldenberg et al., 1995). In vivo studies have shown that antisense oligomers can enter into target organs (Vlassov et al., 1993; Vlassov et al., 1994), and induce changes in target enzymes (Brand et al., 2001; Arora et al., 2002). The transdermal delivery of neutral PMOs using chemical penetration enhancement techniques has yet to be investigated, though preliminary data has demonstrated that the flux of a PMO targeted to cytochrome P4503A2 in propylene glycol and linoleic acid was nearly 20 times greater than that reported with iontophoretic delivery of the same sequence with a phosphorothioate (PS) backbone (Brand and Iversen, 2000). Furthermore, in vivo topical application led to inhibition of cytochrome P4503A2 levels in rat liver and skin (Arora et al., 2002).

The objectives of this study are to investigate the transdermal delivery potential of several PMOs with different sizes, lengths, base compositions, sequences, and lipophilicities as well as to determine the effect of vehicle composition on the permeation of these PMOs through hairless mouse skin. Eleven different PMOs were synthesized complementary to telomeric or biologically relevant gene targets and delivered across hairless mouse skin in vehicles composed of either 95\% propylene glycol, $5 \%$ linoleic acid, water, $50 \%$ water $/ 50 \%$ solution of $95 \%$ propylene glycol, $5 \%$ linoleic acid, and $75 \%$ water $/ 25 \%$ solution of $95 \%$ propylene glycol, $5 \%$ linoleic acid. The effect of vehicle combinations on oligomer penetration will be used to aid in the design of delivery systems for topical or systemic antisense targets.

\section{Material and methods}

\subsection{Synthesis of oligomers}

The PMOs were synthesized at AVI BioPharma (Corvallis, OR) using a Gilson AMS422 Automated Peptide Synthesizer by solid phase synthesis (on a $1 \%$ divinylbenzene crosslinked aminomethylpolystyrene resin) from activated Morpholino subunits, prepared from commercially available ribonucleosides. After synthesis completion, cleavage from resin, and ammonolytic removal of base protecting groups, the trityl-on oligomers were purified by ion exchange (SAX) chromatography on a Bio-Rad EconoSystem low pressure chromatography system. Following purification, a 6-carboxyfluorescein group was attached to the 5'-terminus of the oligomer, which were then detritylated, desalted by reversed phase (RP) chromatography, and lyophilized to yield PMOs in powder form. Analysis of the PMOs was performed by HPLC (SAX and RP) and MALDI-TOF mass spectrometry. The PMOs were then reconstituted in either sterile water or propylene glycol.

\subsection{Octanol/water partition coefficients}

Two milliliters each of water and octanol were placed in glass test tubes, which were then capped and rocked overnight to allow for equilibration. Four hundred microliters of a $5 \mu \mathrm{M}$ oligomer solution was then added to the octanol/water and the tube was rocked for $24 \mathrm{~h}$ (Carelli et al., 1992). At that time, the two phases were allowed to separate and the concentration of oligomer in each phase was determined fluorometrically, with a Turner Quantech fluorometer (Barnstead Thermolyne, Dubuque, IA).

\subsection{Transdermal delivery}

Female hairless mice (CRL: SKH1, Charles River Laboratories, Wilmington, MA) dorsal skin was freshly excised and placed into a modified Bronaugh diffusion chamber system (Permegear, Rieglesville, PA), where the temperature of the skin was maintained at $32{ }^{\circ} \mathrm{C}$ throughout the experiment. The skin was equilibrated in buffer for $3 \mathrm{~h}$ before the beginning of each experiment. One hundred microliters of PMOs, diluted to a final concentration of $5 \mu \mathrm{M}$ in either 95\% propylene glycol, $5 \%$ linoleic acid (PG/LA), water, 50\% water $/ 50 \%$ PG/ LA or $75 \%$ water $/ 25 \%$ PG/LA, were exposed to the epidermal side of the skin. The diffusion chamber's receiver compartment was perfused with $25 \mathrm{mM}$ HEPES, $133 \mathrm{mM} \mathrm{NaCl}$ at $\mathrm{pH} 7.4$ at a flow rate of $2.1 \mathrm{ml} / \mathrm{h}$. Fractions of the effluent were collected in $1 \mathrm{~h}$ intervals. 


\subsection{Assay methods and data analysis}

Oligomer flux was assayed by determining the fluorescence of each fraction with a Turner Quantech fluorometer (Barnstead Thermolyne, Dubuque, IA) set to $490 / 515 \mathrm{~nm}$ (excitation/emission). Because skin auto fluoresces at these wavelengths, background controls were performed with each of the vehicles (PG/LA, water, 50\% water $/ 50 \% \mathrm{PG} / \mathrm{LA}$, or $75 \%$ water $/ 25 \% \mathrm{PG} / \mathrm{LA})$. The receiver solution fluorescence of the control skin was measured at each time point and then subtracted from those determined after delivery of the fluorescently labeled oligomers, so that the differences in fluorescence were due to oligomer flux. This technique was verified by demonstrating that the transdermal absorption of a fluorescently labeled phosphorothioate oligonucleotide targeted to a 6-base telomeric oligomer was the same as an ${ }^{35} \mathrm{~S}$-labeled version of the same sequence (Brand and Iversen, 1996).

The slope of the linear portion of the cumulative transport versus time curve was taken as the steadystate flux. Each oligomer was tested between three and eight times in each of either three or four vehicles $(95 \%$ propylene glycol, $5 \%$ linoleic acid (PG/ LA), water, $50 \%$ water $/ 50 \%$ PG/LA or $75 \%$ water $/ 25 \%$ PG/LA). All values are expressed as mean flux \pm s.e.m. The enhancement ratio for each compound in each vehicle was determined by dividing the flux of a compound in a particular vehicle by its flux in propylene glycol. The mean penetration from PG/LA and water were added together to obtain a theoretical addition, which should be equivalent to the penetration from $50 \%$ PG/LA:50\% water, if there are no synergistic or inhibitory effects. The error associated with this curve was determined by adding the variance of each mean and then finding the standard error from the new variance. Significant differences were assessed via ANOVA followed by a Tukey post-test at the level of $P<0.05$, using GraphPad Prism (GraphPad Software, San Diego, CA).

\subsection{Photomicrography}

Representative skin samples were fixed for further analysis by fluorescent microscopy. Skin was removed from the diffusion chamber, blotted dry, trimmed down so that regions of the skin not exposed to the donor vehicle were cut away, and then placed in plastic molds filled with embedding medium for frozen tissue processing (Sakura Finetek, Torrance, CA). The molds were then wrapped in aluminum foil to prevent exposure to light and frozen in a $-80^{\circ} \mathrm{C}$ freezer. Cryostat sections $(5 \mu \mathrm{m})$ were cut at Oregon State University Veterinary Diagnostic Laboratory (Corvallis, OR). Slides were air-dried in dark for $30 \mathrm{~min}$ and cover-slip mounted using Fluoromount-G mounting medium (Southern Biotechnology Associates, Birmingham, AL). A Nikon Diaphot 300 microscope connected to an Olympus (Melville, NY) Magnafire SP-brand digital camera was used to take the photomicrographs. The exposure times for all fluorescent pictures were kept constant at $11 \mathrm{~s}$.

\section{Results}

Eleven different PMOs were successfully synthesized complementary to telomeric or biologically relevant gene targets. To determine the effect of size and sequence on passive transdermal delivery, the TAG and CTA oligomers were synthesized to various lengths consisting of a base repeat unit with final lengths of $6,9,12,18$ or 24 bases. The telomeric mimic sequence tested ( $\left.5^{\prime}-\mathrm{d}(\mathrm{TTAGGG})-3^{\prime}\right)_{n}$ is denoted as TAG-N and its complements are termed CTA-N (5'-d(CCCTAA)- $\left.3^{\prime}\right)_{n^{\prime}}$ where $n$ corresponds to the number of repeat units in the TAG and corresponding CTA oligomers, and $n$ corresponds to the number of total bases, i.e. TAG-12 has 12 bases consisting of two TTAGGG repeat sequences and CTA24 consists of 24 bases consisting of 4-CCCTAA repeat sequences. All of these lengths were synthesized for both the TAG and CTA oligomers. The large numbers of guanines in the longer TAG molecules resulted in very poor solubility in all solvents, leading to cessation of their use in this study. Four oligomers targeted to biologically active sites were synthesized, termed MYB, MYC-1, MYC-2 and P53. The MYC-1 and MYC-2 oligomers are both directed at the c-myc gene, but differ in their sequence and lipopholicity. These oligomers were used to reveal more broadly applicable information concerning size and sequence. Descriptors of each oligomer, including name, sequence, length, molecular weight, percent guanine composition, and log octanol/water coefficient are summarized in Table 1. 
Table 1. Summary of phosphorodiamidate Morpholino oligomers properties

\begin{tabular}{|c|c|c|c|c|c|}
\hline Oligo name & Sequence & Length $^{\mathrm{a}}$ & $\mathrm{MW}^{\mathrm{b}}$ & $\log K_{\text {ow }}{ }^{c}$ & $\% \mathrm{G}^{\mathrm{d}}$ \\
\hline TAG-6 & 5'_-TTA GGG-3' & 6 & 2686.36 & 0.14 & 50 \\
\hline TAG-9 & 5'-TTA GGG TTA-3' & 9 & 3686.21 & 0.55 & 33 \\
\hline TAG-12 & 5'-TTA GGG TTA GGG-3' & 12 & 4752.1 & 1.20 & 50 \\
\hline CTA-6 & 5'-ССС TTA-3' & 6 & 2566.28 & 0.68 & 0 \\
\hline CTA-12 & 5'-ССС ТТА ССС ТТА-3' & 12 & 4511.95 & 0.46 & 0 \\
\hline CTA-18 & 5'-ССС ТTA ССС ТTA ССС ТTA-3' & 18 & 6457.62 & 0.10 & 0 \\
\hline CTA-24 & 5'-ССС ТТА ССС ТТА ССС ТТА ССС ТТА-3' & 24 & 8403.28 & -0.19 & 0 \\
\hline MYB & 5'-TAT GCT GTG CCG GGG TCT TCG GGC-3' & 24 & 8716.44 & 0.75 & 42 \\
\hline MYC-1 & 5'-ACG TTG AGG GGC ATC GTC GC-3' & 20 & 7378.33 & -0.39 & 40 \\
\hline MYC-2 & 5'-GCT AAC GTT GAG GGG CAT CG-3' & 20 & 7402.36 & 0.02 & 40 \\
\hline P53 & 5'-ССС TGC ТСС ССС CTG GCT CC-3' & 20 & 7106.13 & 0.68 & 15 \\
\hline
\end{tabular}

a. Oligomer length in terms of the number of bases.

b. Molecular weight $(\mathrm{g} / \mathrm{mol})$.

c. Log octanol/water partition coefficient.

d. Percent of guanine bases in oligomer (no. of G's/total number of bases ×100).

PMO penetration across hairless mouse skin was first determined in PG/LA. For both the TAG and CTA molecules, flux was inversely proportional to size $\left(r^{2}=0.94\right.$ for both data sets) (Figure 1, Table 2) and overall CTA oligomers had greater flux than the TAG molecules, for all lengths tested $(P<0.05)$. The biologically active oligomers all have similar size (20 or 24 bases long) and were also passively delivered in $95 \%$ propylene glycol/5\% linoleic acid across hairless mouse skin (Table 2, Figure 2). There was some correlation between size and penetration, as evidenced by the similar transport of the two 24mers, MYB and CTA-24. The two MYC oligomers exhibited higher flux than would be expected based

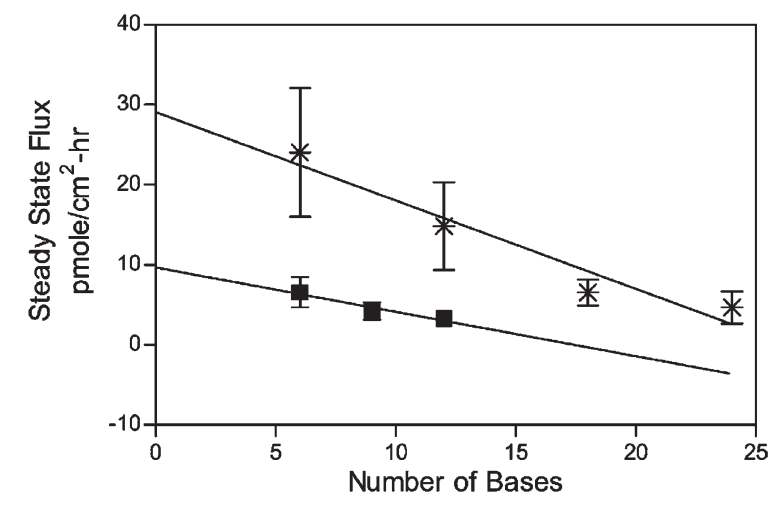

Figure 1. Comparison of size (number of bases) and steady state flux across hairless mouse skin for telomeric compounds in PG/LA ( $\left.{ }^{*}, \mathrm{CTA} ; \mathbf{\mathbf { n }}, \mathrm{TAG}\right)$. Data is reported as mean flux \pm S.E. $\left(r^{2}=0.94\right.$ for both data sets $)$. on their size, and yet even with their different sequences and lipopholicities, they penetrated similarly. The P53 oligomer, however, had lower flux than would be expected based on its size.

To examine the effect of vehicle on PMO delivery, penetration across hairless mouse skin was then determined in water, $50 \%$ water $/ 50 \%$ PG/LA and for three representative oligomers, $75 \%$ water $/ 25 \%$ PG/LA. Mean steady-state flux, standard error, and number of replicates for each of the 11 compounds in all vehicles, as well as the enhancement ratio relative to the PG/LA solvent for each of the vehicles, are summarized in Table 2 . There are a number of possible effects that altering the solvents could have on the transdermal penetration of PMOs including no change in flux, flux inhibition or flux enhancement.

For the TAG molecules, the flux from the water vehicle was $30 \%$ of that obtained with the PG/ LA vehicle (Table 2). The 50\% water/50\% PG/LA vehicle resulted in transport similar to that of just the PG/LA vehicle $(P>0.05)$, and thus demonstrates that a vehicle with even 50\% PG/LA enhances the penetration of the TAG molecules as well as a $100 \%$ PG/LA vehicle. TAG-12 was selected as a representative molecule. Its transport from the $75 \%$ water/25\% PG/LA vehicle was found to be intermediate to that achieved with either just water or just PG/LA (Table 2).

A water vehicle severely inhibited the penetration of the CTA oligomers causing a steady-state 
Table 2. Effect of vehicle composition on transport of oligomers

\begin{tabular}{|c|c|c|c|c|c|c|c|c|c|c|c|}
\hline \multirow[t]{2}{*}{ Oligo name } & \multicolumn{2}{|r|}{$\begin{array}{l}95 \% \text { PG/ } \\
5 \% \text { LA } \\
\end{array}$} & \multicolumn{2}{|c|}{$\begin{array}{c}50 \% \text { PG/LA/ } \\
50 \% \text { water } \\
\end{array}$} & \multicolumn{2}{|c|}{$\begin{array}{c}25 \% \text { PG/LA/ } \\
75 \% \text { water } \\
\end{array}$} & \multicolumn{2}{|c|}{$\begin{array}{l}100 \% \\
\text { water }\end{array}$} & \multicolumn{3}{|c|}{$\begin{array}{c}\text { Enhancement } \\
\text { ratio }^{\mathrm{a}}\end{array}$} \\
\hline & $n^{\mathrm{b}}$ & SS flux ${ }^{c}$ & $n^{\mathrm{b}}$ & SS flux ${ }^{c}$ & $n^{\mathrm{b}}$ & SS flux ${ }^{c}$ & $n^{\mathrm{b}}$ & SS flux ${ }^{c}$ & $50 / 50$ & $25 / 75$ & Water \\
\hline TAG-6 & 8 & $6.6 \pm 1.9$ & 7 & $5.3 \pm 2.0$ & & & 5 & $2.0 \pm 0.9$ & 0.81 & & 0.3 \\
\hline TAG-9-1 & 7 & $4.2 \pm 1.1$ & 6 & $2.6 \pm 1.1$ & & & 5 & $1.2 \pm 0.3$ & 0.62 & & 0.27 \\
\hline TAG-12 & 10 & $3.3 \pm 0.6$ & 7 & $4.2 \pm 1.6$ & 3 & $1.4 \pm 0.1$ & 6 & $1.0 \pm 0.3$ & 1.29 & 1.36 & 0.32 \\
\hline CTA-6 & 6 & $24.1 \pm 8.0$ & 6 & $2.0 \pm 0.4$ & & & 7 & $0.4 \pm 0.2$ & 0.08 & & 0.02 \\
\hline CTA-12 & 7 & $14.8 \pm 5.5$ & 7 & $8.1 \pm 3.4$ & 4 & $1.2 \pm 0.3$ & 8 & $0.2 \pm 0.1$ & 0.55 & 6.87 & 0.01 \\
\hline CTA-18 & 6 & $6.6 \pm 1.6$ & 5 & $7.4 \pm 5.0$ & & & 7 & $0.5 \pm 0.3$ & 1.13 & & 0.07 \\
\hline CTA-24 & 5 & $4.7 \pm 2.0$ & 3 & $0.9 \pm 0.7$ & & & 6 & $0.4 \pm 0.2$ & 0.19 & & 0.08 \\
\hline MYB & 6 & $4.2 \pm 1.0$ & 4 & $15.3 \pm 3.3$ & & & 4 & $3.9 \pm 0.8$ & 3.68 & & 0.93 \\
\hline MYC-1 & 5 & $11.3 \pm 2.8$ & 3 & $15.3 \pm 6.3$ & 3 & $5.1 \pm 1.6$ & 5 & $9.3 \pm 4.3$ & 1.38 & 0.54 & 0.84 \\
\hline MYC-2 & 7 & $10.7 \pm 2.6$ & 3 & $22.1 \pm 4.4$ & & & 5 & $14.3 \pm 4.2$ & 2.07 & & 1.34 \\
\hline P53 & 6 & $2.3 \pm 0.6$ & 3 & $0.0 \pm 0.0$ & & & 6 & $0 \pm 0.00$ & 0 & & 0 \\
\hline
\end{tabular}

a. Mean steady state flux of each oligomer in a particular vehicle divided by its flux in 95\% PG/5\% LA.

b. Number of experimental replications for each vehicle combination.

c. Mean steady state flux in pmole $/ \mathrm{cm}^{2} \mathrm{~h}$ from 5 'M donor solution \pm S.E.

flux of less than $10 \%$ of that achieved with PG/LA for all four CTA oligomers (Table 2). The $50 \%$ water $/ 50 \%$ PG/LA vehicle resulted in transport intermediate to that of water and PG/LA for the CTA oligomers, with the exception of CTA-18, whose transport in the $50 \%$ vehicle was nearly equivalent to its flux in the PG/LA vehicle.

The MYB oligomer delivered in either water or PG/LA had similar transport $(P>0.05)$. However, combining the two vehicles (50\% water/50\% PG/ LA), resulted in a synergistic effect, in which the transport of MYB from the combined vehicle was greater than the sum of the individual effects of the

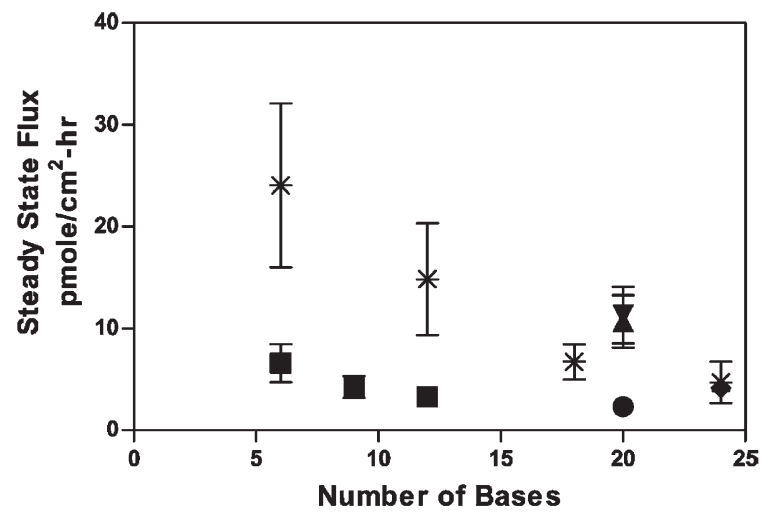

Figure 2. Comparison of number of bases and steady state flux across hairless mouse skin for all oligomers tested in PG/LA (*, CTA; $\bullet$, TAG; •, P53; $\bullet$ MYB; $\boldsymbol{\nabla}$, MYC-1; $\boldsymbol{\Delta}$, MYC-2). Data reported as mean steady state \pm S.E. two vehicles (Table 2, Figure 3). For both of the MYC oligomers, use of a water vehicle resulted in transport similar to that achieved with PG/LA $(P>0.05)$, but combining the two vehicles (50\% water/50\% PG/LA) for either MYC oligomer resulted in an additive effect, that is the sum of the individual effects of the vehicles approximated the transport of the combination of vehicles $(P>0.05)$ (Table 2$)$.

The P53 oligomer exhibited no transport across the skin in any vehicle that contained water and low penetration when applied in PG/LA (Table 2).

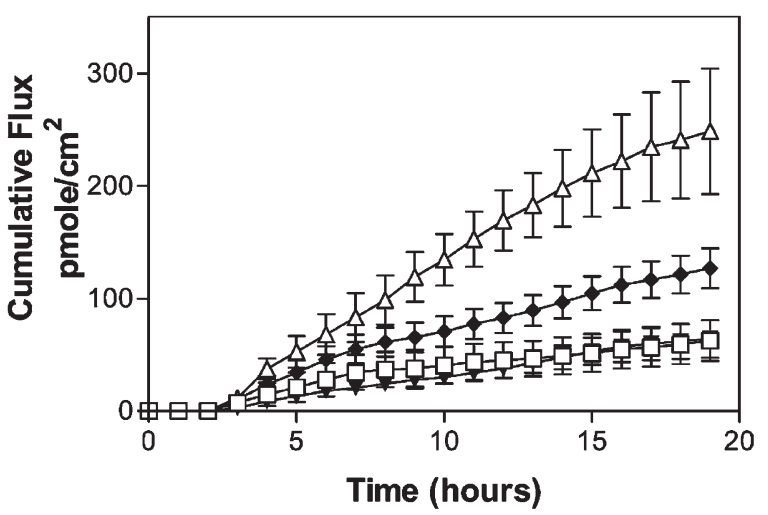

Figure 3. The proposed synergistic effect of vehicle combinations on the skin permeation of the MYB oligomer. Theoretical addition curve was determined by adding the mean of the two individual vehicle cumulative curves, at each time point ( $\square$, PG/LA; $\Delta$, PG/LA:water; $\nabla$, water; $\bullet$ theoretical addition). S.E.M. for the theoretical curve was found by adding the individual variances and then converting to S.E.M. 


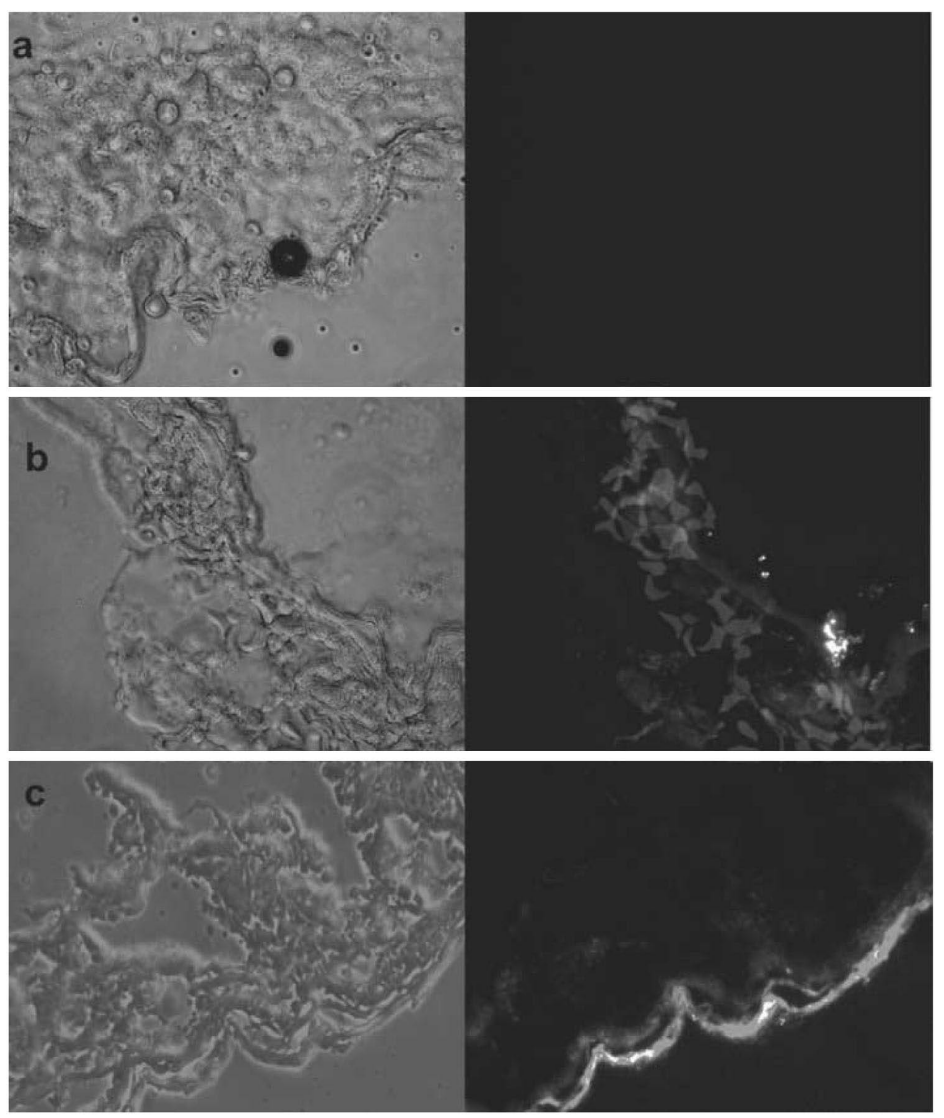

Figure 4. Comparison of the phase contrast and fluorescence photomicrographs of (a) control skin, (b) P53 oligomer delivered in PG/LA, (c) P53 oligomer delivered in a water vehicle. Note lack of fluorescence of control skin and that oligomer reaches keratinocytes when given in PG/LA, but remains on top of skin when applied in water (magnification 200×).

This flux data is supported by three sets of photomicrographs demonstrating that the majority of the compound remained on top of the stratum corneum, with minimal localization within the skin when applied in water. In contrast, P53 applied in PG/LA showed penetration into the skin and accumulation in the keratinocytes which would account for smaller than expected transdermal flux. The untreated control demonstrates that the fluorescence is due to the oligomer and not from the skin itself (Figure 4).

Examination of the penetration data revealed no correlation between flux and log octanol/water coefficient for any vehicle. PMOs with few guanines have their transdermal penetration inhibited in the presence of water (CTA and P53); however, the presence of G-quartets (MYB, MYC-1 and MYC-2) prevents this decrease in absorption.

\section{Discussion}

This work was designed to begin elucidating the relationship between PMO characteristics and appropriate selection of chemical penetration enhancers. The data demonstrate that size, sequence, and thus base composition, will influence the transdermal flux of uncharged biologically active PMOs. Furthermore, modifying the concentration of the model penetration enhancer, propylene glycol, altered the absorption of the PMOs through the skin in a sequence specific manner.

Several PMOs with biologically relevant targets were selected for these studies. Telomeres are stretches of a repeated sequence (5'-TTAGGG-3') which form a cap at the end of the chromosome and are essential for chromosome protection and stability. After each round of DNA replication the telo- 
meric portions of chromosomes are progressively shortened due to incomplete replication of 5 '-ends of the linear DNA by the DNA polymerases. Telomerase is an enzyme that can add 6-base repeats units of telomeric DNA to the 3'-ends of chromosomes, replacing the loss of telomeric DNA that occurs at each cell division. While telomerase is present in only a limited range of normal adult cells, studies have demonstrated that telomerase activity is activated in up to $90 \%$ of all human cancers, and may be important in their immortalization (Urguidi et al., 1998). Inhibition of telomerase represents an ideal therapy against tumor growth. Since most normal cells lack this enzyme, it is an appealing target, in that, inhibition could cause minimal damage elsewhere in the patient. Previously, the ability of the phosphorothioate TAG oligomers to specifically inhibit telomerase activity (Mata et al., 1997) and permeate the skin, using iontophoresis (Brand and Iversen, 1996; Brand et al., 1998a) has been demonstrated.

The other oligomers studied were antisense to some biologically relevant targets, including p53, c$m y b$ and c-myc. p53, a tumor suppressor gene, regulates the activity of several cell-cycle related genes and is important in cell proliferation. Mutations of this gene are found in more than half of all human cancers, including skin cancers, and the expression of p53 is up-regulated after ultraviolet radiation damage to DNA (Levine, 1997). The protooncogene c-myc is a transcriptional activator that plays a critical role in multiple pathways leading to the control of normal growth, cell cycle progression, cell proliferation, differentiation and apoptosis. It is expressed in nearly all replicating cells and expression is reduced after terminal differentiation. Elevation of this transcriptional activator and the DNA binding protein, c-myb, is associated with several pathologies, including restinosis and carcinogenesis (Nasi et al., 2001; Kipshidze et al., 2001).

Propylene glycol is one of the most widely used penetration enhancers in dermatological formulations and has previously been shown to enable the absorption of topically applied PMOs completely through the skin (Arora et al., 2002). It can however lead to skin irritation at concentrations greater than $50 \%$ (v/v) (Lashmar et al., 1989). The ability of vehicles consisting of lower concentrations of propylene glycol to enhance the permeation of oligomers was therefore investigated. Propylene glycol func- tions as a cosolvent, by accumulating in the stratum corneum, thereby changing its solubility characteristics. It acts synergistically to increase partitioning of candidate molecules and other enhancers such as fatty acids into the stratum corneum, thus significantly increasing the activity of co-enhancers (Walters, 1989; Barry, 1991; Hadgraft et al., 1992; Asbill et al., 2000). Fatty acids, such as linoleic acid, serve as penetration enhancers by disrupting ceramidecholesterol and or cholesterol-cholesterol ordered structures in the stratum corneum, leading to increase drug permeation (Asbill et al., 2000).

The transport of the PMOs mimicking both the telomeres (TAG oligomers) and their complements (CTA oligomers) was examined in order to isolate the effect of either size or sequence on transdermal absorption. For both the TAG and CTA oligomers, transport across the skin was inversely related to their size when applied in a PG/LA formulation. This would be expected based on the "rules" governing transdermal drug delivery (Kasting et al., 1987). In a similar study (Brand et al., 1998a) using iontophoretic delivery of phosophorothioate oligomers with the TAG and CTA sequences, the general inverse trend between size and flux was seen, but the linearity was not as clear as for the PG/LA studies presented in this work. A similar relationship between size and flux was also identified for passively delivered methylphosphanate oligomers (Nolen et al., 1994) and iontophoretically delivered phosphodiester oligomers with equal portions of each base (Oldenberg et al., 1995). However, the effects of size and sequences, as well as oligomer chemistry and penetration techniques, cannot be separated in these previous studies. Interestingly, the size-flux trend was not evident for either the TAG or CTA sequences when the vehicle was changed to $50 \%$ PG/LA:50\% water or $100 \%$ water.

The power of synthesizing two series of oligomers (TAG and CTA) with increasing sequence length is that it enables the separation of the effects of size versus sequence on transdermal penetration for the PMOs. This approach is similar to studies examining the percutaneous absorption of the alkanols ranging from methanol to nonanol (Durrheim et al., 1980). While size was linearly related to transdermal transport for the TAG and CTA molecules, there are obvious differences in penetration due to sequence. 
Examining the other oligomers in this study reveals some diverse effects of size on transdermal permeation. The MYB oligomer exhibited flux similar to what would be expected in terms of its size, given that its transport was equal to that of CTA24. The P53 oligomer, which was the same size as the MYC oligomers, exhibited substantially lower flux. The explanation for this P53 observation, however, was provided by examining the fluorescent photographs that demonstrated its extensive localization within the keratinocytes when delivered in the PG/LA vehicle.

As oligomers cross the skin, the first viable cells they encounter are the keratinocytes. Keratinocytes are very responsive to oligomers, because they can internalize these molecules rapidly, without cell surface accumulation or endosomal sequestration (Noonberg et al., 1993; Nestle et al., 1994). The MYC-1 and P53 results are consistent with earlier studies that correlated the delivery of PSOs across hairless mouse skin with their uptake by keratinocytes (Brand et al., 1998b). They found that a PS MYC oligomer, with the same sequence as the PMO MYC-1 studied here, had almost no keratinocyte uptake but had very high transport across the skin, consistent with the transport of the MYC1 oligomer in this study. Additionally the PS P53 oligomer, again with the same sequence as the one in this study, had very high uptake by keratinocytes, with low transport across the skin, identical to the findings in this paper.

While molecular size can impact transdermal delivery, lipophilicity is actually considered more important than size in predicting the transdermal penetration of small molecules (Potts and Guy, 1992). Two different sequences (MYC-1, MYC-2) were synthesized, targeted to inhibit expression of the c-myc gene. We hypothesized that these oligomers would have different penetration levels because their lipophilicities differ by a factor of four. These oligomers both had a higher flux than would be expected based on their size, but similar flux to each other, indicating that lipophilicity does not seem to play a role in their absorption.

The different transdermal delivery potential of the TAG and CTA molecules may be explained by the large number of guanines present in the TAG oligomers. These bases may cause the oligomers to fold into structures that are not easily transported through the skin (Smith and Feigon, 1993). Studies on iontophoretic transport of the TAG-9 PSO revealed that subtle changes in molecular structure can have a significant impact on the percutaneous penetration of oligomers. Modifying the center of the molecules reduced polarity and steric-hindrance while the mass/charge ratio increased, resulting in greater penetration (unpublished results). Furthermore, comparing the iontophoretic transdermal penetration of a 15-base thrombin aptamer consisting of nine guanines and six thymidines with a globular three-dimensional (3D) structure to a scrambled aptamer without a similar 3D structure revealed that the active compound had two-fold lower penetration than the scrambled sequence, again demonstrating the influence of structure on transdermal delivery (Oldenberg et al., 1995). The effects of guanines on dermal penetration can also be seen when water is used as a solvent. Flux was significantly inhibited in those sequences that contained low levels of guanine residues (CTA \& P53), was not effected at all for those PMOs containing a G-quartet (MYC-1, MYC-2 and MYB) and was partially inhibited for the PMOs with only three G's in a row (TAG).

Comparing the transport of all oligomers in this study with that achieved by their counterpart phosphorothioate oligomers delivered with iontophoresis indicates the TAG molecules, delivered in any vehicle, can achieve permeation similar to that achieved with iontophoresis (Brand et al., 1998a). MYC-1 and MYC-2 oligomer transport in all vehicles was nearly the same as that achieved with iontophoresis, with MYC-2's transport in the $50 \%$ propylene glycol (with linoleic acid) $/ 50 \%$ water vehicle exceeding that achieved with iontophoresis. Finally, MYB transport was higher in all vehicles in this study (water, 50\% water/50\% PG/LA, and PG/LA) than with iontophoresis, with transport in the $50 \%$ water $/ 50 \%$ PG/LA being over 10 times that achieved with iontophoresis.

In summary, for sequences targeted to the enzyme telomerase, flux is related inversely to size, supporting previous studies which report similar results for other oligomer chemistries (Nolen et al., 1994; Brand et al., 1998a; Oldenberg et al., 1995). Additionally, differential transport of equivalently sized oligomers of varying sequence suggests that 
oligomer permeation is sequence specific. While sequence and size can be used to explain most transport, the MYC and P53 oligomers reveal that the target for these antisense molecules may also play a role in determining the ability of these compounds to cross the skin with some oligomers (TAG-6) being better for transdermal delivery and others (P53) for topical applications. Furthermore, the PMO sequence will influence the selection of an optimal penetration enhancer with those containing Gquartets having good transport in a water containing solvent. This study also has demonstrated that PMOs can achieve passive transport across skin that meets or surpasses that of iontophoretically delivered PSOs of equivalent sequence.

\section{Acknowledgments}

The authors would like to acknowledge Anna Charron, Adam Pont, and Drs. David Jones and Susan Cuppett for their assistance with this work. Additionally, we thank Tim Sirois for his advice on statistical analysis. This material is based upon work supported under a National Science Foundation Graduate Research Fellowship (AKP).

\section{References}

Arora, V., Hannah, T. L., Iversen, P. L. and Brand, R. M., 2002. Transdermal use of phosphorodiamidate Morpholino oligomer AVI-4472 inhibits cytochrome P450 3A2 activity in male rats. Pharm. Res. 19, pp. 1465-1470.

Asbill, C. S., El-Kattan, A. F. and Michniak, B., 2000. Enhancement of transdermal drug delivery: chemical and physical approaches. Crit. Rev. Ther. Drug Carrier Syst. 17, pp. 621-658.

Barry, B., 1991. The LPP theory of skin penetration enhancement. In: Bronaugh, R. L., Maibach, H. J. (Eds. ), In vitro Percutaneous Absorption: Principles, Fundamentals, and Applications. CRC Press, Boston, pp. 165-186.

Brand, R. M. and Iversen, P. L., 1996. Iontophoretic delivery of a telomeric oligonucleotide. Pharm. Res. 13, pp. 851-854.

Brand, R. M. and Iversen, P. L., 2000. Transdermal delivery of antisense compounds. Adv. Drug Deliv. Rev. 44, pp. 51-57.

Brand, R. M., Wahl, A. and Iversen, P. L., 1998. Effects of size and sequence on the iontophoretic delivery of oligonucleotides. J. Pharm. Sci. 87, pp. 49-52.
Brand, R. M., Haase, K., Hannah, T. L. and Iversen, P. L., 1998. An experimental model for interpreting percutaneous penetration of oligonucleotides that incorporates the role of keratinocytes. J. Invest. Dermatol. 111, pp. 1166-1171.

Brand, R. M., Hannah, T. L., Norris, J. and Iversen, P. L., 2001. Transdermal delivery of antisense oligonucleotides can induce changes in gene expression in vivo. Antisense Nucleic Acid Drug Dev. 11, pp. 1-6.

Carelli, V., Di Colo, G., Nannipieri, E. and Serafini, M. L., 1992. Enhancement effects in the permeation of Alprazolam through hairless mouse skin. Int. J. Pharm. 88, pp. 89-97.

Durrheim, H., Flynn, G. L., Higuchi, W. I. and Behl, C. R., 1980. Permeation of hairless mouse skin I: Experimental methods and comparison with human epidermal permeation by alkanols. J. Pharm. Sci. 69, pp. 781-786.

Hadgraft, J., Walters, K. A. and Guy, R. H., 1992. Epidermal lipids and topical drug delivery. Semin. Dermatol. 11, pp. 139-144.

Hudziak, R. M., Barofsky, E., Barofsky, D. F., Weller, D. L., Huang, S. and Weller, D. D., 1996. Resistance of Morpholino phosphorodiamidate oligomers to enzymatic degradation. Antisense Nucleic Acid Drug Dev. 6, pp. 267-272.

Kasting, G., Smith, R., Cooper, E., 1987. Effect of lipid solubility and molecular size on percutaneous absorption. In: Shroot, B., Schaefer, H., Karger, S. (Eds. ), Skin Pharmacokinetics. Basel, pp. 128-153.

Kipshidze, N., Moses, J., Shankar, L. R. and Leon, M., 2001. Perspectives on antisense therapy for the prevention of restenosis. Curr. Opin. Mol. Ther. 3, pp. 265-277.

Lashmar, U. T., Hadgraft, J. and Thomas, N., 1989. Topical application of penetration enhancers to the skin of nude mice: a histopathological study. J. Pharm. Pharmacol. 41, pp. 118-121.

Levine, A. J., 1997. p53, the cellular gatekeeper for growth and division. Cell 88, pp. 323-331.

Mata, J. E., Joshi, S. S., Palen, B., Pirruccello, S. J., Jackson, J. D., Elias, N., Page, T. J., Medlin, K. L. and Iversen, P. L., 1997. A hexameric phosphorothioate oligonucleotide telomerase inhibitor arrests growth of Burkitt's lymphoma cells in vitro and in vivo. Toxicol. Appl. Pharmacol. 144, pp. 189-197.

Nasi, S., Ciarapica, R., Jucker, R., Rosati, J. and Soucek, L., 2001. Making decisions through myc. FEBS Lett. 490, pp. 153-172.

Nestle, F. O., Mitra, R. S., Bennett, C. F., Chan, H. and Nickoloff, B. J., 1994. Cationic lipid is not required for uptake and selective inhibititory activity of ICAM-1 phosphorothioate antisense oligonucleotides in keratinocytes. J. Invest. Dermatol. 103, pp. 569-575. 
Nolen III, H. W., Catz, P. and Friend, D. R., 1994. Percutaneous penetration of methyl phosphonate antisense oligonucleotides. Int. J. Pharm. 107, pp. 169-177.

Noonberg, S. B., Garovoy, M. R. and Hunt, C. A., 1993. Characteristics of oligonucleotide uptake in human keratinocyte cultures. J. Invest. Dermatol. 101, pp. 727-731.

Oldenberg, K. R., Kham, T., Smith, G. A. and Selick, H. E., 1995. Iontophoretic delivery of oligonucleotides across full thickness hairless mouse skin. J. Pharm. Sci. 84, pp. 915-921.

Potts, R. O. and Guy, R. H., 1992. Predicting skin permeability. Pharm. Res. 9, pp. 663-669.

Smith, F. W. and Feigon, J., 1993. Strand orientation in the DNA quadruplex formed from the Oxytricha telomere repeat oligonucleotide $\mathrm{d}(\mathrm{G} 4 \mathrm{~T} 4 \mathrm{G} 4)$ in solution. Biochemistry 32, pp. 8682-8692.

Stein, D., Foster, E., Huang, S. -B., Weller, D. and Summerton, J., 1997. A specificity comparison of four antisense types: Morpholino, 2'-O-methyl RNA, DNA, and phosphorothioate DNA. Antisense Nucleic Acid Drug Dev. 7, pp. 151-157.
Summerton, J. and Weller, D., 1997. Morpholino antisense oligomers: design, preparation and properties. Antisense Nucleic Acid Drug Dev. 7, pp. 187-195.

Urguidi, V., Tarin, D. and Goodison, S., 1998. Telomerase in cancer: clinical applications. Ann. Med. 30, pp. 419-430.

Vlassov, V. V., Karamyshev, V. N. and Yakubov, L. A., 1993. Penetration of oligonucleotides into mouse organism through mucosa and skin. FEBS Lett. 327, pp. 271-274.

Vlassov, V. V., Nechaeva, M. V., Karamyshev, V. N. and Yakubov, L. A., 1994. Iontophoretic delivery of oligonucleotide derivative into mouse tumor. Antisense Res. Dev. 4, pp. 291-293.

Walters, K. A., 1989. Penetration enhancers and their use in transdermal therapeutic systems. In: Hadgraft, J., Guy, R. H. (Eds. ), Transdermal Drug Delivery. Marcel Dekker, New York, pp. 197-246. 